\title{
Research on Strength Quality Training Methods of the Examinee Major in Aerobics
}

\author{
Zhenghong $\mathrm{Li}^{1} \mathrm{a}^{*}$, Lin $\mathrm{Li}^{\mathrm{b}}$ and Lei Zhang ${ }^{1, \mathrm{c}}$ \\ ${ }^{1}$ Sports and Science College, Jishou University Renmin South Road 120, Jishou City Hunan \\ Province, China \\ a316225603@qq.com, b2862923557@qq.com, c448716587@qq.com
}

Keywords: Aerobics; Special strength; Training methods; Examinee

\begin{abstract}
The power is essential physical quality requirements for the athletes in the sports, which is the same important for the examinee major in Aerobics. There are static and dynamic actions about difficult action aerobics. To complete the two kinds of difficulty actions needs athletes to have a certain power as support. This paper, based on the characteristics of aerobics and the factors that affect the examinee performance, using the methods of literature method, logic reasoning method and so on, and drew the lessons from the previous research on strength training methods and analyzed it and summarized some scientific systematical special strength training methods, so as to provide some suggestions and methods to guide the training of examinee major in aerobics.
\end{abstract}

\section{Introduction}

Aerobics, as rhythmic gymnastics class project, fully demonstrates such movement characteristics as balance, strength, flexibility, coordination and so on. The series of difficulty actions in complete sets of movements are determined by athletes' strength quality [1]. Professional strength capacity is also very important for examinees to improve their examination results. In training practice, it is often seen that the increase of examinees' strength is riotously out of proportion to the increase of their examination results. The examinees' strength improved, but they can't combine with the technical characteristics of aerobics events, which make the examinees, have strength vainly and cannot apply it to the event. So, their examination results also not necessarily can be improved. We can't just to the increase of the strength of the examinee. We should pay more attention to apply the strength to the aerobics technique. Only in this way, can we improve the achievement of examinee. So, we should choose some special strength training method scientifically.

\section{Research Methods}

Literature Method. This paper, by consulting books and papers of China Hownet, reading a large number of literatures about the special strength training, which provide a large amount of data to the research of this paper, compares with some of the training methods and summarizes a set of specific training methods.

Expert Interviewing Method. Through the analysis of the research of some experts, the paper summarized a set of reasonable and training methods of combining various training methods, to ensure the improvement of examinees' examination results.

Inductive Analysis Method. Organized and scientifically analyzed the various data and information collected, summarized and studied them through judgment reasoning and logical thinking process.

\section{The Difference between General and Special Strength on Examinees Major in Aerobic}

When examinees train in the peacetime, the work of muscle group is different [2]; Differences are: one is to do isometric contraction, the other is to do plyometric contraction. When the muscles do plyometric contraction, the efficiency of the muscles can be improved. In specific practice, 
muscles take on plyometric contraction. This is also the difference between the two. Not all the practice methods are suitable for the examinees. So, we should lay down special training methods that accords with examinees according to the difference between the two. Both can improve the strength. But, on the premise of improving the examinee's scores, combined with special characteristics, make the training methods combine with them.

\section{Analysis of Strength Quality Training Methods and Means of Examinees Major in Aerobics}

Analysis of Special Strength Quality Training Methods and Means of Upper Limbs of Examinees Major in Aerobics. There are plenty of methods about upper limb training. They are: the bench pressing and holding the dumbbell and swinging arms. The muscles' strength of the Examinees' upper limbs, chests and shoulders can be reflected through bench press. In order to improve the ability of upper limb muscles, the ways and means of bench press with barbell can make the examinees get good effect. Gradually increase the training load, improve the examinees' ability to quickly complete the set of action. [3].

Analysis of Special Strength Quality Training Methods and Means of Trunk of Examinees Major in Aerobics. Body strength training is very important for all sports. It not only develop part of the strength. At the same time, is very important for the power of whole body and the coordinated ability. The ability has the close relationship with special qualities. So, the key points are to train the trunk. Trunk strength training methods are: the Examinee can enhance the balance of the body and the strength of the trunk through practice single flat foot (or single hand) support. Practice with moderate intensity, hands are as wide as shoulder. The palms purport the ground below the shoulder. The upper limbs, trunk and lower limbs are in one line. And then, raise one hand or one foot and keep for one minute and 30 seconds (to adjust time according to the examinee's ability). Do four to six groups altogether. Each of groups gradually increases the intensity and decreases the time of each group. All in All, the coaches should adjust the corresponding training contents according to the training target. And pointedly adopt the training methods and plans conform with them .[4] Of course, coaches can adopt some training methods related to some aerobics technique at last. Using some exercise combination can practice coordination ability of examinees' upper and lower limbs and waist and back muscles. Due to the technical requirements for aerobics exercise, muscle coordination between ability is necessary, we can use different ways to practice ability. Seen from aerobics technical action, in the process of completing the whole set of movements, the examinees need to tighten up their waist, back, so as to demonstrate their physical beauty and reach the maximum potential energy. Therefore, we must strengthen the torso exercise of power, which can make examinees improve the technology, and thus enhance the examination performance of examinees. [5]

Analysis of Special Strength Quality Training Methods and Means of Lower Limbs of Examinees Major in Aerobics. Except for a good examinee has good upper body strength, lower limb strength is not ignored either. Examinee can improve the strength of lower limbs by such research methods as squat, jump and standing long jump and so on. The squat is to improve muscle strength of the thigh. The power of lower refers to the ability to work each other between different muscles of lower limbs. In the process of sports teaching, the most convenient, effective and simple traditional test method is the vertical jump and standing long jump. The two indicators have great correlation with the power of lower limbs. The related research shows that whether the vertical jump or standing long jump can reflect the ability of quick contraction of examinees' muscles. In order to enhance the power of examinees' lower limb muscles, so, when practice, examinees can combine with special technology, further improve their special level.[6]

The Demands for the Strength Quality of competitive aerobics competition rules to the Examinees Major in Aerobics. Firstly, The Demands for the Balance of Complete Choreography.

In the process of creating complete set of movements of competitive aerobics, most of them require examinees to fully show their style characteristics and skill levels, make the complete set of actions have rich artistic appeal. The cohesion of all the actions in the complete set have no fault and be finished perfectly. The selection of difficulty action in the complete set of movements has 
three levels of combination: In the air, standing and on the ground. The set of contents must be in line with their own theme, and require the perfect combination of music and movement and body, so bring the mental pictures to the audiences and referees. The difficulty movement groups of competitive aerobics in the complete set are divided into four groups: A, B, C and D. There are 10 elements of difficulty. They cannot be repeated. There is at least one element of difficulty in each group, otherwise it will be regarded as the lack of group [7]. According to <2013-2016 aerobics competition rules>, considering the balance of every difficulty in the complete set. Only develop examinees' quality of strength systematically and comprehensively, can they complete the whole complete set of movements. Secondly, The Marking Requirements of Completion Situation. There is specific requirements in <2013-2016 aerobics competition rules $>$ : Whether action is standard or not is judged through examinee's posture, which demands that examinee keep upper body upright, head lifting high, belly tighten and hips clamped and the middle of body tighten. In the process of complete sets of movements, the mutual coordination of limbs of the body is inseparable from the good physical quality [8]. And the most important point is that, also the characteristics of aerobics being different from other forms of dance, it is required when complete a set of action, it is not only requires large movement range, but also clearly see the beginning and the end. In another word, it is the brake. Brake needs certain power quality as the base. The brake is restricted by the strength. Therefore, the function of strength quality in the complete set of movements has not been ignored.

The Problems the Examinees Major in Aerobics should pay attention to about Special Strength Quality Training. Firstly, Special Strength Training Combining with Specialized Training

Specialized training is to train specifically certain parts of the body. The special strength training is to practice based on event characteristics and technical essentials[9]. On the basis of which muscles are working when aerobics examinees are moving, we can use the special training to improve working muscles strength alone and to meet the requirement of examinee for the strength. We are also on the basis of power generation characteristics of aerobics, make specific training combine with specialized training, thus improve the achievement of examinee. When complete the set of movements, it not only needs strength, it also needs speed. They turn the kinetic energy into potential energy as much as possible. So, they should improve their own speed as much as possible. We can not only improve the strength of the part of body, but also comprehensively improve the special strength of whole body. Secondly, The Coordinated Development of Special Strength Quality and other Quality Training. In the sports, the main factors of aerobics examinees' competitive ability include the competitive strengths and the ability to give full play to their professional knowledge. Competitive strength, is crucial in the competitive ability. Competitive strength is made up of physical strength, skill strength, tactics strength, power strength and other factors. As is known to all, the competitive level of examinees occupies the main position in the form factor of the competitive ability. Physical ability includes strength quality. And strength quality is the foundation of other quality [10]. Examinees' special grade is determined by many factors. Taking physical quality as example, speed, coordination, agility, flexibility and other factors play an important role to improve special performance. Aerobics is also such. It not only needs strength, but also these qualities are indispensable and all the qualities are improved. Examinees should be combined with various factors in the usual special training so as to raise the special level. Thirdly, Deal with Different Examinee in Different Ways. When carry on special strength training, each of the examinees should be dealt in different ways. Because each of examinees is a separate individual, they are different in different stages and different situations. Their gender, age and body quality are also different. So, act appropriately to the situation and draw up the special strength training methods. If the strength training can be combined with the actual situation in the process of training, the effect will be better. [11] 


\section{Conclusion}

Aerobics is a type of sport that presents strength and beauty, which has specialized special strength training methods. Special strength training should have a clear plan and is in accordance with its special characteristics and technology of the examinees. And now, our aerobics special strength training methods aren't perfect enough. In the usual training process, the coaches don't tell the difference between general strength training and special strength training, make the examinees to take on single, one-sided special training, Finally, the characteristics of the aerobics examinees' special strength training should be recognized and develop with other quality coordinately and deal with different examinee in different ways. When carry on special strength training, each of the examinees should be dealt in different ways. Because each of examinees is a separate individual, they are different in different stages and different situations.

\section{Acknowledgements}

This research was financially supported by2015Western philosophy and Social science Fund project of Hunan province(5YBX037) Sports Science Society Project of Hunan Province (2016 xh037); 2015Philosophy and Social Science Fund Project of Hunan province (15YBA318); 2015Scientific Research Key project of Hunan Province Department of Education (15A154); 2014 Core Curriculum group Construction Projects of General Class of Jishou University.

\section{References}

[1] Zhang Tong, Li Xuemei, On the Importance of the Strength Quality Training to Examinees Major in Aerobics, Golden Field,Vol.315, 2014, pp.474.

[2] Wei Ankui, Effect of Strength Training on Specific Performance, Vol.28, No.3, 2004, pp.45-48.

[3] Xie Huisong, Zhou Tieming, Comparative Study of Different Levels of Special Event Strength for Our Talent Women People -Vaulting Players, Vol.34, No.12, 2008, pp.53-57.

[4] Xu yue. The Investigation and Analysis of the Present After-class Training Situation of Track and Field Class of Higher Vocational and Technical College in Xinjiang [D]. Xinjiang normal university, 2010.

[5] Shen Hongxing, Analysis of Trunk and Neck Strength Characteristics of Excellent Greco-roman Wrestling Athletes of Shandong Province, Shandong Sports Institute, 2009.

[6] Chai Jianwei, Research on the Correlation between the Shijiazhuang City Youth $1000 \mathrm{M}$ Rowing Examinee Special Strength and Performance [D]. Hebei normal university, 2015.

[7] Sun Peng, Li Shichang, Wang Hong, Comparative Analysis of Difficult Movements of World Competitive Aerobics, Vol.4, 2010, pp.55.

[8] Chen Zongdi, Li Chengxi, Wang Ming, The Multi-factor Analysis of

[9] How to Develop the Aerobics Expressiveness, Journal of hubei sports technology, Vol.3, 2004, pp.355.

[10]Wang Fudong, Qi Pengyu, Discussion of Special Strength Training for Sprint Team of Daqing Sports School, The Harbin sports institute journal, Vol.3, 2008, pp. 111.

[11]Nie Huimin, Research on the Curriculum Design and Implementation of < Sports Quality Training > Physical Fitness Professional Direction in Sports Colleges and Universities, Beijing Sport University, 2006.

[12] Wang Lili, Research on the Framework of Training Contents of Examinees Major in Aerobics Aged from 15 to 17 in China, Suzhou University, 2009. 